\title{
Cold and Warm Gas Outflows in Radio AGN
}

\author{
Raffaella Morganti ${ }^{1,2}$, Joanna Holt ${ }^{3}$, \\ Clive Tadhunter ${ }^{4}$, and Tom Oosterloo ${ }^{1,2}$ \\ ${ }^{1}$ Netherlands Foundation for Research in Astronomy, \\ Postbus 2, 7990 AA, Dwingeloo, The Netherlands \\ Email: morganti@astron.nl \\ ${ }^{2}$ Kapteyn Astronomical Institute, University of Groningen, \\ P.O. Box 800, 9700 AV Groningen, The Netherlands \\ ${ }^{3}$ Leiden Observatory, Leiden University, PO Box 9513, 2300 RA Leiden, The Netherlands \\ ${ }^{4}$ Department of Physics and Astronomy, University of Sheffield, Sheffield, S3 7RH, UK
}

\begin{abstract}
The study of the conditions and the kinematics of the gas in the central region of AGN provides important information on the relevance of feedback effects connected to the nuclear activity. Quantifying these effects is key for constraining galaxy evolution models. Here we present a short summary of our recent efforts to study the occurrence and the impact of gas outflows in radio-loud AGN that are in their first phase of their evolution. Clear evidence for AGN-induced outflows has been found for the majority of these young radio sources. The outflows are detected both in (warm) ionized as well in (cold) atomic neutral gas, and they are likely to be driven (at least in most of the cases) by the interaction between the expanding jet and the medium. The mass outflow rates of the cold gas (H I) appear to be systematically higher than those of the ionized gas. The former reach up to $\sim 50 M_{\odot} \mathrm{yr}^{-1}$ and are in the same range as "mild" starburst-driven superwinds in ULIRGs, whilst the latter are currently estimated to be a few solar masses per year. However, the kinetic powers associated with these gaseous outflows are a relatively small fraction (a few $\times 10^{-4}$ ) of the Eddington luminosity of the galaxy. Thus, they do not appear to match the requirements of the galaxy evolution feedback models.
\end{abstract}

Keywords. ISM: jets and outflows, galaxies: jets, galaxies: ISM, galaxies: active, radio lines: galaxies

\section{Introduction}

Relativistic plasma jets represent one of the possible ways in which the enormous energy released by an active nucleus can interact with and affect the interstellar medium. In powerful radio sources, the kinetic power of such jets is comparable to the energy released via radiation emission or winds and, therefore, it is important to consider the impact that this can have on the life and evolution of the host galaxy. Quantifying this impact is necessary in order to set tighter constraints on the effects of feedback from AGN. Indeed, due to a lack of such observational constraints, feedback is often invoked as a "black box" and often relies on powerful AGN winds (see, e.g., Di Matteo et al. 2005; Hopkins et al. 2005). Whilst this may be true for radio-quiet AGN, it is likely that in radio-loud AGN the expanding radio jets also contribute to the feedback via jet-induced outflows.

Different diagnostics have already clearly shown that, on scales of tens to hundreds of kiloparsecs, radio emission can have an impact on the ISM (e.g., on its kinematics and possibly ionization and morphology). For example, in many powerful radio galaxies, the extended emission lines show highly perturbed kinematics in the regions along the radio axis (see some of the nearby examples shown by, e.g., Clark et al. 1997, Villar-Martín et al. 
1999, and Solórzano-Iñarrea et al. 2001). In high- $z$ radio galaxies, high velocity gradients are observed in the Ly $\alpha$ halos (see, e.g., Villar-Martín et al. 2001 and references therein). In some cases, the presence of fast gas outflows $\left(>1000 \mathrm{~km} \mathrm{~s}^{-1}\right)$ has been confirmed through integral field spectroscopy (see Nesvadba et al. 2006, 2009). Extreme examples of interaction between the radio plasma and ISM are the X-ray cavities in the hot gas that are hollowed out by expanding radio lobes (see, e.g., McNamara \& Nulsen 2007). The existence and the characteristics of such cavities show that the radio emission can prevent the hot gas from cooling and forming stars. These studies already illustrate that the impact of radio plasma jets is not only limited to their (often) very collimated structures, but that the effect extends to the surrounding gas by the jet impinging on the ISM. This perturbed, wide region is also seen in the results of numerical simulations (see, e.g., Krause et al. 2009 and references therein for some recent examples) showing how the passage of the jet creates a large cocoon of shocked ambient gas.

This interaction is likely to be even more relevant during the early stages of the evolution of the radio source, i.e., when the jet is interacting with the natal cocoon of gas deposited in the inner regions during the triggering event, often considered to be a merger and/or interaction (see, e.g., Bicknell, Dopita, \& O'Dea 1997). Figure 1 illustrates examples of the evolution with time of the highly complex interplay between a newly born radio jet and the rich and clumpy surrounding ISM. These are obtained from the numerical simulations of Geoff Bicknell and collaborators (see Sutherland \& Bicknell 2007 and Saxton et al. 2005 and references therein).

This short summary presents our recent efforts to quantify, using observations of ionized and neutral atomic hydrogen, the characteristics of the gas in the nuclear regions of young radio sources and the occurrence and the impact of gas outflows (and in particular jet-driven outflows) connected to the first phase of the evolution of their radio jets.

\section{Young Radio Sources: The Ionized Gas ...}

Newly born or young radio sources are identified by their small linear size combined with steep radio spectra. They are usually dubbed "gigahertz-peaked" or "compact steep spectrum" sources (see Fanti et al. 1995 and O'Dea 1997 for reviews). We have used a sample of 14 powerful, young radio sources (Holt et al. 2008, 2009) to investigate, from the very beginning of their evolution, the impact of the radio plasma on the surrounding gas. Young radio sources are known to often live in a gas-rich medium as expected if a

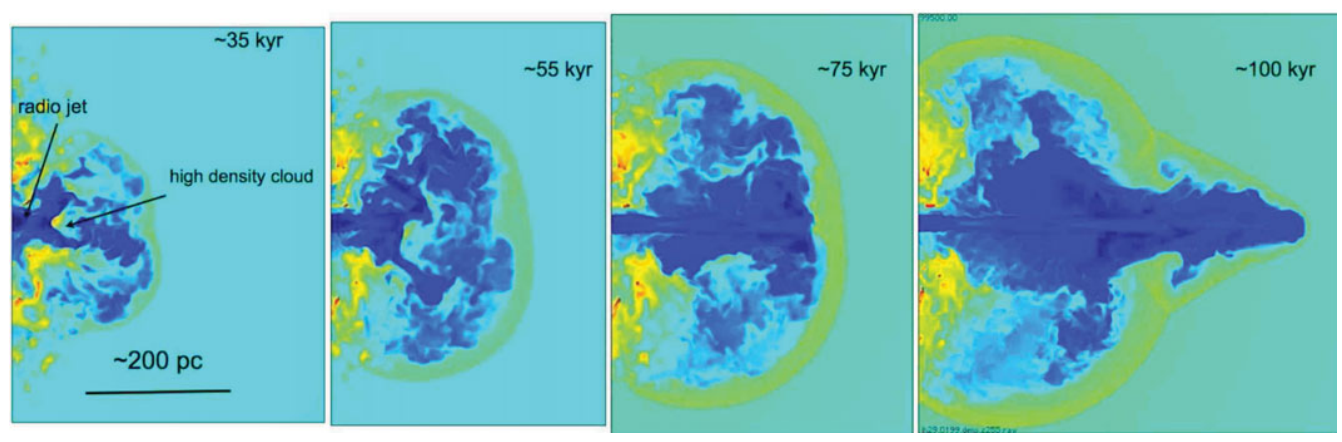

Figure 1. Snapshots of numerical simulations illustrating, at different times, the evolution of the interaction of a plasma jet with an inhomogeneous, clumpy medium (Sutherland \& Bicknell 2007; Wagner \& Bicknell in preparation). 
merger or interaction has provided the triggering fuel for the AGN (see, e.g., de Vries et al. 1999; Axon et al. 2000; Vermeulen et al. 2003; Morganti et al. 2005). Indeed, this is further supported by our recent results, which show that both the ionized gas and neutral atomic hydrogen ( $\mathrm{H} \mathrm{I}$ ) are commonly observed in these sources.

In the case of the ionized gas, the observed emission lines have been modeled in order to investigate the kinematics and ionization properties of the gas (see Holt, Tadhunter, \& Morganti 2008, 2009 for details). To enable accurate line modeling, the continuum (comprised of a derived nebular continuum and a modeled stellar continuum) was subtracted. This modeling has also allowed us to investigate the stellar populations in these sources, yielding particularly interesting results (Holt et al. 2007). Most of these young radio sources contain a young $(<0.5 \mathrm{Gyr}$ to down to a few Myr) stellar population that accounts for a significant fraction of the stellar mass $(\sim 10-50 \%)$. This further suggests that these sources have gone through a gas-rich merger event in their recent past and this event is likely responsible for both the burst of star formation and the triggering of the AGN.

We find that the ionized gas often extends well beyond the radio source (up to $\sim 20 \mathrm{kpc}$ ). These halos usually have quiescent kinematics (sometimes suggesting that the gas is in rotation), in agreement with the fact that they have not been affected by the passage of the radio source. The presence of such halos is shown in Figure 2 and Figure 3 in the cases of PKS 1549-79 and PKS 1345+12, respectively. Thus, we have used the kinematics of the quiescent halos of ionized gas to derive the systemic velocity of the host galaxy. Deriving a reliable systemic velocity has been a key step in the interpretation of the kinematics of the gas (both warm and cold).

On the other hand, the emission lines in the nuclear regions are typically very complex (confirming earlier findings, e.g., Gelderman \& Whittle 1994), requiring multiple Gaussian components to model them. Furthermore, the majority of the objects studied show
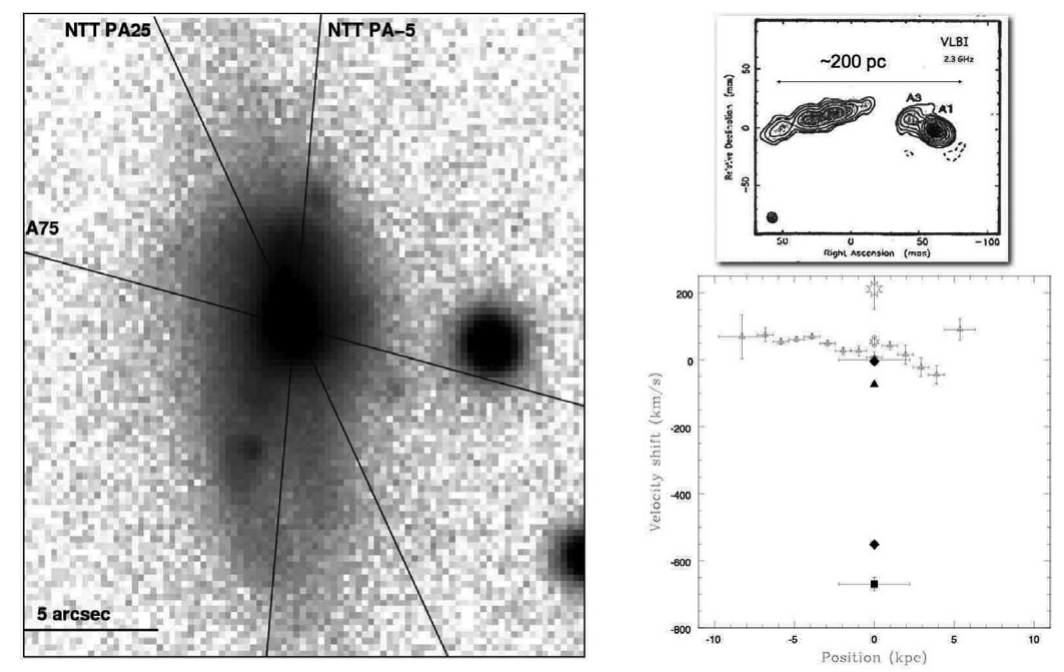

Figure 2. Left: Deep VLT $r$-image of PKS1549-79 ( $\left.5^{\prime \prime}=12 \mathrm{kpc}\right)$. The slit positions used for the long-slit spectroscopic observations are shown for reference. Right top: VLBI $2.3 \mathrm{GHz}$ image of PKS 1549-79. The stronger component represents the radio core. Right bottom: Radial velocity profiles of PKS 1549-79 obtained from the slit position PA-5. Small open and filled triangles represent the narrow and intermediate components of $\mathrm{H} \alpha$ respectively. Overplotted is the radial velocity of the deep H I $21 \mathrm{~cm}$ absorption (large filled triangle at $-30 \mathrm{~km} \mathrm{~s}^{-1}$ ). See Holt et al. (2006) for more details. 
that the broader components tend to be blueshifted compared to the systemic velocity, suggesting nuclear outflows. The shifts are up to $2000 \mathrm{~km} \mathrm{~s}^{-1}$ (see, e.g., PKS $1345+12$ in Figure 3, Holt et al. 2003). These results show that the presence of fast gas outflows is (almost) ubiquitous in these objects. Although limited by small number statistics, Holt et al. (2008) noted that the characteristics of these fast gaseous outflows appear to be also related to the size and to the orientation (with respect to the line of sight) of the source. In particular, the most extreme of these nuclear outflows are in the smaller gigahertz-peaked sources (with linear size $<1 \mathrm{kpc}$ ).

As a final remark before moving to the characteristics of the cold gas, it is interesting to note that the hot component of the gas also shows signatures of strong interactions between the radio jets/lobes and the ISM. For example, Chandra has revealed how even the lowest luminosity radio sources will be initially overpressured, expanding supersonically and shock heating their host galaxy ISM (e.g., Heinz et al. 1998). These effects are observed in a number of objects (see, e.g., Jetha et al. 2008, Croston et al. 2007, and Massaro et al. 2009), the most famous being Centaurus A (Kraft et al. 2003; Croston et al. 2009).

\section{3. ... and the $\mathrm{HI}$}

$\mathrm{HI}$ absorption against the strong radio continuum is often detected in the nuclear regions of radio galaxies, but it is even more common in powerful, young radio sources (see also Vermeulen et al. 2003). Although the detection of H I absorption gives only a limited view of the distribution of the cold atomic gas (being limited to the regions in front of the radio continuum), it still represents a sensitive tool to detect gas with column densities down to $N_{\mathrm{HI}} \sim 10^{19} \mathrm{~cm}^{-2}$, much lower than what can be traced with $\mathrm{H} \mathrm{I}$ in emission, especially in distant objects. Different structures can produce the observed H I absorption (e.g., circumnuclear disks, infalling clouds perhaps connected with the fueling of the AGN, outflowing gas, or large-scale disks seen in projection against the nuclear regions of the galaxy). In order to disentangle which of these structures is actually responsible for the absorption, it is crucial to combine the $\mathrm{H}$ I results with those from the ionized gas, particularly for establishing a reliable systemic velocity.

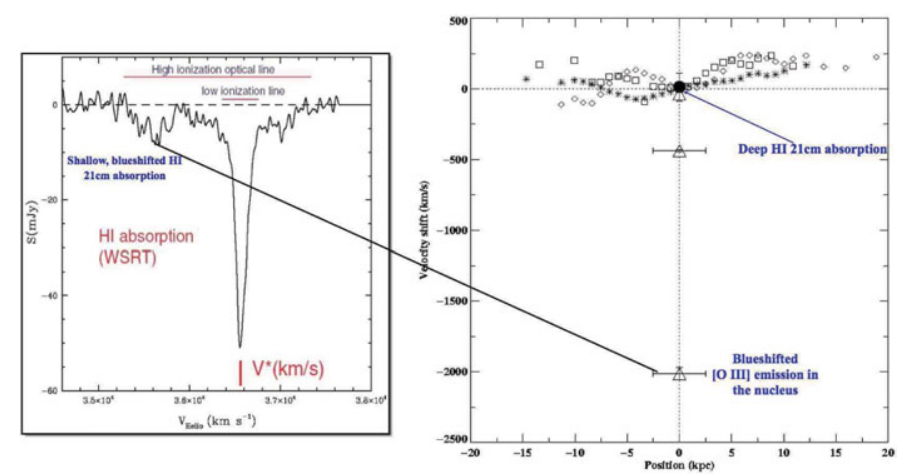

Figure 3. Left: H I profile showing a deep absorption close to the systemic velocity and a broad blueshifted wing ranging more than $1000 \mathrm{~km} \mathrm{~s}^{-1}$. Right: Radial velocity profiles of PKS 1345+12. For more details, see Holt et al. (2003). Overplotted is the radial velocity of the deep H I $21 \mathrm{~cm}$ absorption, and the location of the blueshifted absorption is also indicated. 
A surprising result in the study of $\mathrm{H}$ I in young radio sources has been the discovery of fast H I outflows (Morganti et al. 2003, 2005a and references therein). These outflows are characterized by the detection of broad, blueshifted $\mathrm{H}$ I absorption profiles (see, for example, the case of PKS 1345+12 in Figure 3 and of 3C 305 in Figure 4). The velocities of these outflows exceed, in the most extreme cases, $1000 \mathrm{~km} \mathrm{~s}^{-1}$. Such high velocities were not expected to be associated with cold gas. The optical depth of the outflowing H I gas component is typically quite low, therefore making these absorption structures particularly difficult to detect with present-day radio telescopes.

It is also difficult to identify the location of the fast outflowing gas. This is the main limitation if we want to understand their origin. Various possible outflow driving mechanisms include starburst superwinds, quasar-induced winds, and expanding radio jets. Most of the outflows are limited to the central regions ( $<$ a few $\mathrm{kpc}$ ), i.e., co-spatial with the radio source. This rules out the wide starburst wind as the origin (see, e.g., Batcheldor et al. 2007 for more details). The radio/optical alignment and the co-spatial location between the outflows in ionized and neutral gas and the radio structure (in particular radio hot spots) is instead a strong indication that expanding radio jets could be a more likely mechanism. Indeed, it is interesting to see that in the few cases where the location of the H I outflow has been identified, it is off-nucleus and coincident with regions where the jet appears to strongly interact with the surrounding medium. One of the best cases is the radio galaxy 3C 305 (Morganti et al. 2005b), shown in Figure 4. The broad H I absorption is found $\sim 1.6 \mathrm{kpc}$ off-nucleus at the location of the northeast radio lobe. This is the same location where an outflow of ionized gas occurs (Jackson et al. 1995).

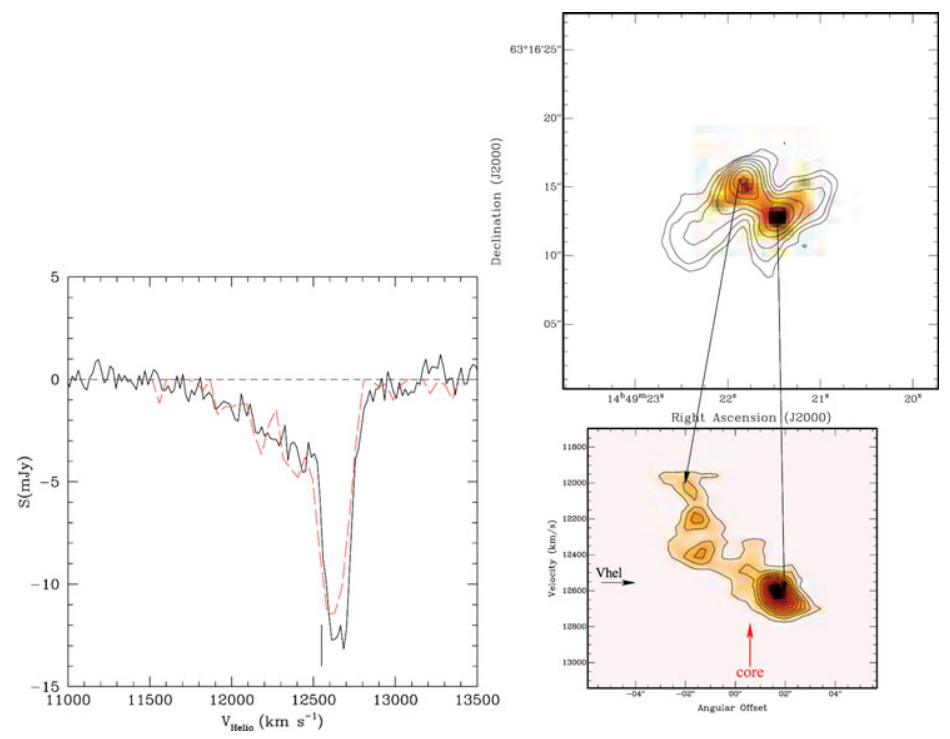

Figure 4. Left: H I absorption profile obtained with the WSRT (solid line) superimposed on the integrated spectrum from the high-spatial resolution VLA observations (long-dashed). The profile shows a deep, relatively narrow, absorption and a broad component that covers more than $1000 \mathrm{~km} \mathrm{~s}^{-1}$ at zero intensity, mostly blueshifted compared to the systemic velocity indicated by the vertical solid line. Right: Panel showing the radio continuum image in contour and the integrated H I absorption (grey scale) from the VLA data (top) and the position-velocity plot from a slice passing through the two lobes (bottom). The gray scale image represents the total intensity of the H I absorption, see Morganti et al. (2005b) for details. 
Further support for this hypothesis comes from the Chandra detection of hot, X-ray emitting gas (Massaro et al. 2009) that has been interpreted as thermal emission due to the interaction between matter outflows and shock-heated environment gas. The fact that the two phases of the gas (cold neutral and warm ionized) show similar kinematics suggests that these outflows are produced by the same mechanism. This is a recurrent characteristic in all the best-studied cases.

The characteristics and the location of the outflow in 3C 305 - but also in other cases like 3C 293 and IC 5063 (Morganti et al. 2007) — strongly suggests that the outflow is produced by the interaction between the radio jet and the ISM.

As a final remark, it is important to note that the presence of cold gas associated with the observed fast outflows indicates that the gas can cool efficiently after a strong interaction, as predicted by numerical simulations of jets impacting on gas clouds (Mellema et al. 2002; Fragile et al. 2004).

\section{Implications}

Fast gaseous outflows of ionized and neutral atomic gas (broad blueshifted H I absorption) are extremely common in young, powerful radio sources. In addition, we also note that recently restarted radio sources (e.g., 3C 236 and 3C 293) show similar characteristics. Interestingly, all the objects with fast gaseous outflows typically have a particularly rich ISM; molecular gas $\left(\mathrm{CO}\right.$ and $\mathrm{H}_{2}$ ) as well as PAH lines detected from Spitzer IRS spectra appear to be more common in these young sources than in large radio galaxies (Dicken et al., in preparation). These characteristics support the idea that these objects have recently been triggered (or re-triggered) by a gas-rich merger/accretion, and the newly born (or re-born) radio jet is now fighting its way through the medium deposited by this event.

Puzzling in this respect is the result from the detailed study of the line ratios of the ionized gas presented by Holt et al. (2009). This study shows that the ionization of these lines is not dominated by shocks, as one would have expected from their kinematics. While densities and temperatures derived from the broad emission-line components (i.e., the components that are more kinematically disturbed by the passage of the radio plasma) are typically high, as expected in the presence of fast shocks, Holt et al. (2009) were unable to unambiguously distinguish the dominant ionization mechanism using the optical emission-line ratios.

If gas outflows are so ubiquitous in young, powerful radio sources, what is the impact they have on the evolution of the host galaxy? The results and the picture sketched above are consistent with the idea that the interaction between the radio plasma and the ISM would actually help clear the central regions of material deposited during the event (e.g., interaction/merger) that could have triggered both AGN and starbursts. In this phase, the black hole is also rapidly growing (close to the Eddington rate), while the AGN is obscured by the large concentration of dust and gas. The effect of the outflows should therefore be to remove gas from the central regions and to halt both star formation and further accretion. According to the models, this would require that $\sim 5-10 \%$ of the accretion power of the AGN drives the winds/outflows (Di Matteo et al. 2005; Hopkins et al. 2005).

It is, therefore, interesting to see what is the mass of the gas outflow that we observe in the young radio galaxies studied so far. From the best-studied case PKS 1549-79 (see Holt et al. 2006), we derive for the ionized gas:

- Mass of ionized gas in the outflow: $1.9 \times 10^{4} M_{\odot}<M_{\text {outflow }}<1.9 \times 10^{6} M_{\odot}$. 
- Mass outflow rate: $0.12<\dot{M}<12 M_{\odot} \mathrm{yr}^{-1}$.

- Energy flux: $5.1 \times 10^{40} \mathrm{erg} \mathrm{s}^{-1}<\dot{E}<5.1 \times 10^{42} \mathrm{erg} \mathrm{s}^{-1}$.

- Kinetic power: $1.6 \times 10^{-6}<\dot{E} / L_{\text {edd }}<1.6 \times 10^{-4}$.

From these numbers, it is clear that only a small fraction of the accretion power drives the warm outflow in PKS 1549-79 (Holt et al. 2006). Thus, despite the evidence for rapid outflows in the warm gas, the estimated kinematic power in the warm outflow is several orders of magnitude less than required by the feedback models. One possible explanation for this apparent discrepancy is that much of the mass of the AGN-induced outflow is tied up in cooler or hotter phases of the interstellar medium.

The H I component of the outflows appears to be systematically more prominent:

- Mass of neutral gas in the outflow: $\sim 10^{6} M_{\odot}$.

- Mass outflow rate: up to $\sim 50 M_{\odot} \mathrm{yr}^{-1}$.

These parameters place the neutral outflows in the same range as "mild" starburstdriven superwinds in ULIRGs (Rupke et al. 2002). However, also in the case of H I outflows, the ratio between the kinetic powers associated with these gaseous outflows and the Eddington luminosity of the galaxy is still only about a few $\times 10^{-4}$ and therefore relatively low compared to that required by galaxy evolution feedback models.

\section{Conclusions}

The studies done so far clearly show evidence for AGN-induced outflows on all scales in radio galaxies and in particular in young, powerful radio sources. We find that in young (or restarted) radio galaxies $(<5 \mathrm{kpc}$ scale) there is almost ubiquitous evidence for nuclear warm outflows, but they are much less powerful than required by feedback models for bulge evolution. Massive neutral outflows are also detected; these are more massive, comparable to the "mild" starburst-driven winds detected in ULIRGs. These outflows may be common in powerful radio sources, but they are difficult to detect with the sensitivity of present-day radio telescopes. The likely origin of (at least some of) these outflows is interaction between the expanding jets and ISM. Much of the power in AGN-induced outflows may be associated with hotter or cooler phases of the ISM.

As mentioned above, the radio part of these studies is now limited by the sensitivity of present-day radio telescopes. Fortunately, many new radio facilities are planned or are in the process of becoming available. Ultimately, the Square Kilometer Array (SKA, Lazio 2009) will provide a major step forward by allowing a complete census of H i in radio sources (including the weak ones!), detailed studies of the kinematics of the gas close to the nucleus, and the possibility of looking for outflows in weaker sources (Morganti et al. 2004).

In the shorter term, other upgraded or new facilities e.g., EVLA, ASKAP (Johnston et al. 2008), MeerKat (Booth et al. 2009), and Apertif (focal plane array upgrade of the WSRT, Oosterloo et al. 2009) will soon allow an improvement of our knowledge of the $\mathrm{H}$ I characteristics in radio-loud sources.

Furthermore, the LOw FRequency Array (LOFAR, de Vos et al. 2009; Morganti et al. 2009) that is now coming on-line and will operate between 10 and $200 \mathrm{MHz}$, will allow detections of relic structures (also in galaxies that are not currently radio loud!), thus providing insight on the duty cycle of radio activity. 


\section{References}

Axon, D. J., Capetti, A., Fanti, R., Morganti, R., Robinson, A., \& Spencer R. 2000, AJ, 120, 2284

Batcheldor, D., et al. 2007, ApJ, 661, 70

Bicknell, G., Dopita, M. A., \& O'Dea C. P. 1997, ApJ, 485, 112

Booth, R. S., de Blok, W. J. G., Jonas, J. L., \& Fanaroff, B. 2009, MeerKAT Key Project Science, Specifications, and Proposals [arXiv:0910.2935]

Clark, N. E., et al. 1997, MNRAS, 286, 558

Croston, J. H., Kraft, R. P., \& Hardcastle, M. J. 2007, ApJ, 660, 191

Croston, J. H., et al. 2009, MNRAS, 395, 1999

de Vries, W. H., O'Dea, C. P., Baum, S. A., \& Barthel, P. D. 1999, ApJ, 526, 27

de Vos, M., Gunst, A. W., \& Nijboer, R. 2009, in Proceedings of the IEEE, Vol. 97, p. 1431

Di Matteo, T., Springel, V., \& Hernquist, T. 2005, Nature, 433, 604

Emonts, B. H. C., et al. 2006, A\&BA, 454, 125

Fragile, P. C., Murray, S. D., Anninos, P., \& van Breugel, W. 2004, ApJ, 604, 74

Gelderman, R. \& Whittle, M. 1994, ApJS, 91, 491

Heinz, S., Reynolds, C. S., \& Begelman, M. C. 1998, ApJ, 501, 126

Holt, J., Tadhunter, C. N., \& Morganti, R. 2003, MNRAS, 342, 227

Holt, J., et al. 2006, MNRAS, 370, 1633

Holt, J., et al. 2007, MNRAS, 381, 611

Holt, J., Tadhunter, C. N., \& Morganti, R. 2008, MNRAS, 387, 639

Holt, J., Tadhunter, C. N., \& Morganti, R. 2009, MNRAS, 400, 589

Hopkins, P. F., Hernquist, L., Cox, T. J., Di Matteo, T., Martini, P., Robertson, B., \& Springel, V. 2005, ApJ, 630, 705

Inskip, K. J., Villar-Martín, M., Tadhunter, C. N., Morganti, R., Holt, J., \& Dicken, D. 2008, MNRAS, 386, 1797

Jackson, N., Sparks, W. B., Miley, G. K., \& Macchetto, F. 1995, A\&AA, 296, 339

Jetha, N. N., Hardcastle, M. J., Ponman, T. J., \& Sakelliou, I. 2008, MNRAS, 391, 1052

Johnston, S., et al. 2008, Experimental Astronomy, 22, 151

Kraft, R. P., et al. 2003, ApJ, 592, 129

Krause, M. \& Gaibler, V. 2009, in The Interface between Galaxy Formation and AGN, ed. V. Antonuccio-Delogu \& J. Silk, in press [arXiv:0906.2122]

Lazio, J. 2009, in Panoramic Radio Astronomy, in press [arXiv:0910.0632]

Massaro, F., et al. 2009, ApJ, 692, L123

McNamara, B. R. \& Nulsen, P. E. J. 2007, ARAA, 45, 117

Mellema, G., Kurk, J. D., \& Rottgering, H. 2002, A\&A 395, 13

Morganti, R., et al. 2003, ApJ, 593, 69

Morganti, R., Greenhill, L. J., Peck, A. B., Jones, D. L., \& Henkel, C. 2004, New Astron. Rev., 48,1195

Morganti, R., Tadhunter, C. N., \& Oosterloo, T. 2005a, A\& A, 444, L9

Morganti, R., Tadhunter, C. N., Oosterloo, T., van Moorsel, G., \& Emonts, B. 2005b, A\&A, 439,521

Morganti, R., Holt, J., Saripalli, L., Oosterloo, T., \& Tadhunter, C. 2007, A\&A, 476, 735

Morganti, R., et al. 2009, in Panoramic Radio Astronomy: Wide-Field 1-2 GHz Research on Galaxy Evolution, ed. G. Heald \& P. Serra, in press

Nesvadba, N. P. H., et al. 2006, ApJ, 650, 693

Nesvadba, N. P. H. 2009, The Interface between Galaxy Formation and AGN, ed. V. AntonuccioDelogu and J. Silk, in press [arXiv:0906.2900]

O'Dea, C. P. 1998, PASP, 110, 493

Oosterloo, T., et al. 2009, in Widefield Science and Technology for the SKA, in press [arXiv:0912.0093]

Rupke, D. S., Veilleux, S., \& Sanders, D. B. 2002, ApJ, 570, 588

Saxton, C. J., Bicknell, G. V., Sutherland, R. S., \& Midgley, S. 2005, MNRAS, 359, 781 
Solórzano-Iñarrea, C., Tadhunter, C., \& Axon, D. 2001, MNRAS, 323, 965

Sutherland, R. S. \& Bicknell, G. V. 2007, ApJS, 173, 37

Vermeulen, R. C., et al. 2003, A\&SA, 404, 861

Villar-Martín, M., Tadhunter, C., Morganti, R., Axon, D., \& Koekemoer, A. 1999, MNRAS, 307,24

Villar-Martín, M., et al. 2001, MNRAS, 328, 848 